\title{
HIV-I Drug Resistance and Genetic Transmission Networks Among MSM Failing Antiretroviral Therapy in South China 2014-2019
}

\author{
Yun Lan* \\ Xizi Deng* \\ Linghua $\mathrm{Li}$ \\ Weiping Cai \\ Junbin $\mathrm{Li}$ \\ Xiaoli Cai \\ Feng Li \\ Fengyu $\mathrm{Hu}$ (D) \\ Chunliang Lei \\ Xiaoping Tang \\ Guangzhou Eighth People's Hospital, \\ Guangzhou Medical University, \\ Guangzhou, 510030, People's Republic of \\ China \\ *These authors contributed equally to \\ this work
}

Correspondence: Chunliang Lei; Xiaoping

Tang

Guangzhou Eighth People's Hospital,

Guangzhou Medical University, 627

Dongfeng East Road, Yuexiu District,

Guangzhou, 510030, People's Republic of

China

Email gz8hlcl@|26.com; tangxp@gzhmu. edu.cn
Background: Guangdong, located in South China, is one of the areas heavily affected by HIV-1 in China. The transmission of HIV-1 among men who have sex with men (MSM) has gradually been increasing in Guangdong.

Objective: To investigate the characteristics of the HIV-1 drug resistance, and genetic transmission networks in MSM with antiretroviral therapy (ART) failure from 2014 to 2019 in Guangdong.

Methods: HIV-1 pol gene sequences were amplified. An online subtyping tool was used to determine the genotype, and a maximum likelihood phylogenetic tree was reconstructed to confirm the genotype results. The Stanford University HIV Drug Resistance Database was used to analyse the sequences of drug resistance mutations (DRMs) and drug resistance profiles. A pairwise Tamura-Nei 93 genetic distance-based method was used to analyse the genetic transmission networks.

Results: Of 393 sequences isolated from HIV-infected MSM with ART failure, CRF01_AE (47.3\%), CRF07_BC (21.4\%) and CRF55_01B (21.4\%) were the top three strains. 55.2\% individuals harboured NRTI DRMs, whereas 67.4\% carried NNRTI DRMs. 96.8\% cases harboured mutations resistance to NRTIs or NNRTIs at high-level. The most common DRMs were M184I/V (42.2\%), followed by V179D/E (37.9\%) and K65R (27.2\%). Of the subtype B sequences, no sequence fell into a cluster. Of the CRF01_AE, CRF55_01B, and CRF59_01B sequences, $14.5 \%, 61.9 \%$, and $33.3 \%$ fell into clusters, respectively. Of the CRF07_BC sequences, 39.3\% fell into clusters. The majority of MSM in transmission networks were concentrated at age below 35 years old, with multiple links. Moreover, approximately $54.8 \%$ of MSM had more than 2 potential transmission partners.

Conclusion: Drug resistance mutations more frequently occurred in NNRTIs among MSM with ART failure in Guangdong Province. Transmission network analysis revealed a complex transmission pattern, and more attention should be given to younger HIV1-infected MSM with multiple links.

Keywords: HIV-1, drug resistance, genetic transmission networks, MSM

\section{Introduction}

Up to Aug 31, 2018, approximately 850 thousand people living with HIV were reported nationwide in China. ${ }^{1}$ The prevalence of HIV-1 infection is still a massive challenge to public health, although highly active antiretroviral therapy (ART) has improved the quality of life of those living with HIV or AIDS and decreased mortality rates. ${ }^{2}$ Guangdong, located in South China, is one of the areas in China 
that is most heavily affected by HIV-1. The number of people receiving ART has increased since the nationwide National Free Antiretroviral Treatment Program (NFATP) started in the early 2000s in Guangdong Province. HIVinfected people receiving ART have a lower risk of HIV transmission. ${ }^{3}$ However, increasing HIV drug resistance occurs after the expansion of ART and the Treat-all strategy.

Men who have sex with men (MSM) continue to be a major cause of new infections in America, Europe, Oceania, and Asia, ${ }^{4}$ including China. The proportion of newly reported HIV/AIDS cases infected via MSM increased continuously, from $0.7 \%$ in 2005 to $25.8 \%$ in 2014. ${ }^{5}$ In Guangdong Province, MSM with HIV increased from $5.0 \%$ in 2008 to $11.4 \%$ in 2013 of the infected population. ${ }^{6}$ The risk factors for HIV transmission in MSM included multiple sex partners, unprotected anal intercourse, low rates of condom use, drug use, and a history of other sexually transmitted diseases. ${ }^{7}$ More attention should be paid to MSM population, including the circulating HIV strains.

Here, we focused specifically on the occurrence and characteristics of HIV-1 drug resistance mutations (DRMs) and the extent of changes in drug susceptibility with long-term treatment among ART-experienced MSM adults in Guangdong Province, China, by targeting the polymerase ( $p o l)$ gene. The results will help clarify HIV-1 genetic diversity and the progression of drug resistance among ART-failing MSM in Guangdong Province between 2014 and 2019.

\section{Materials and Methods}

\section{Study Population and Data Collection}

HIV-infected patients attending NFATP were regularly followed up at sentinel hospitals in 21 prefectures of Guangdong Province. The plasma HIV-1 viral load after 6 months of ART administration was quantified at least once per year at Guangzhou Eighth People's Hospital. Samples with a viral load of $\geq 1000$ copies/ $\mathrm{mL}$ were subjected to drug resistance genotyping using an in-house RT-PCR method. Patients were eligible for inclusion in this study, including MSM living in Guangdong, followed up from Jan 1st 2014 to Dec 31st 2019, and had a plasma viral load $\geq 1000$ copies $/ \mathrm{mL}$. Patients were excluded if they had used ART outside national guidelines or were missing treatment regimen information.
The epidemiological and demographic information of patients was downloaded and recorded from the National Information Surveillance System, including age, occupation, education level, marital status, WHO classification, geographical region, $\mathrm{CD} 4+\mathrm{T}$ cell count, sampling area and therapeutic regimen.

\section{Nucleic Acid Extraction, Amplification, and Sequencing}

Viral RNA was extracted from $140 \mu \mathrm{L}$ of plasma using a QIAamp Viral RNA Mini Kit (Qiagen, Germany). The amplification of the HIV-1 pol gene fragments (HXB2 2253-3318, covering the full-length protease and the first 240 amino acids of reverse transcriptase codons) was performed using one-step reverse transcription PCR (RTPCR) by using the PrimeScript one-step RT-PCR Kit Ver. 2 (Takara, China), followed by nested PCR using $E x$ Taq Hot Start Version DNA Polymerase (Takara, China). The amplified PCR products were electrophoresed on a $1.0 \%$ agarose gel with the DL2000 DNA marker (Takara, China). The positive products were purified using the Agarose Gel DNA Extraction Kit (Takara, China), according to the manufacturer's protocol and sent to Tianyi Huiyuan Genomics Company for Sanger's sequencing.

\section{Sequence Assembly and Phylogenetic Analyses}

The obtained sequences were assembled and cleaned by DNA sequence analysis software Sequencher V5.4.6 [Gene codes, US.], and the alignments were performed by BioEdit V7.2.

To determine the genotype, the sequences were submitted to the online subtyping tool COMET developed by the Luxembourg Institute of Health (https://comet.lih.lu/). ${ }^{8}$ Then, phylogenetic analysis was conducted to confirm the genotype results.

The alignments, including all the obtained sequences, were merged with HIV-1 subtyping references retrieved from Los Alamos HIV Sequence Database using BioEdit software. Then, the maximum likelihood (ML) tree was reconstructed using PhyML software V3.0 ${ }^{9}$ based on the GTR substitution model. The approximate likelihood ratio test (aLRT) ${ }^{10}$ was used to estimate the branch support. The final trees were visualized using Figtree V1.4.2. The SH-like support aLTR value of 0.9 was selected as the threshold for high reliability of lineages. 


\section{Drug Resistance Analyses}

The HIVdb program from Stanford University HIV Drug Resistance Database (https://hivdb.stanford.edu/hivdb/bysequences/) was used to analyse the sequences for DRMs. ${ }^{11}$ DRMs were classified into the PI, NRTI or NNRTI drug classes. Drug resistance was divided into five levels, and sequences with a low-level or greater category of resistance were defined as drug resistance.

\section{HIV-I Genetic Transmission Network Construction}

To avoid biases due to the potential for convergent evolution driven by antiretroviral therapy, 43 codons in PR and RT associated with DRMs were removed according to the major HIV-1 DRMs last updated on Oct $23,2020 .^{12}$

The pairwise Tamura-Nei 93 (TN93) nucleotide substitution model ${ }^{13}$ was used to calculate the genetic distance for the different genotype sequences analysed using the HyPhy software V2.2.4. The genetic transmission network was visualized and analysed using the network software Cytoscape V3.2.1 with a pairwise genetic distance of $0.005-0.015 .^{14}$ The genetic distance threshold is defined as the distance that identifies the maximum number of clusters in the transmission networks.

\section{Nucleotide Sequence Data}

The nucleotide sequences were submitted to GenBank under the accession numbers MW852088-MW852480.

\section{Statistical Analysis}

An Excel database was established, and all statistical analyses were performed using IBM SPSS V25.0. Qualitative statistics were described using frequency, and quantitative statistics were described using the median (IQR). Categorical variables were compared using Fisher's exact tests. Variables for drug resistance with a P-value $<0.05$ in the Fisher's exact tests were included in the multivariate logistic regression analysis. For all statistical tests, the level of significance for the evaluation of the two-sided $\mathrm{P}$ values was set at 0.05 .

\section{Results}

\section{Characteristics of the Study Population}

Of all the patients who received ART for more than 6 months in Guangzhou Eighth People's Hospital from 2014 to 2019 , with once HIV-1 viral load testing per year, a total of 3895 individuals had a plasma viral load of $\geq 1000$ copies/ $\mathrm{mL}$. Among the 3895 plasma samples, 3584 HIV pol gene sequences were obtained. A total of 393 sequences were from HIV-infected MSM, accounting for $11.0 \%(393 / 3584)$.

The demographic characteristics of these HIV-infected MSM are shown in Table 1. The participants were aged between 18 and 67 years, with a median age of 32 years,

Table I Demographic Characteristics of 393 MSM-Infected Patients with Virologic Failure in ART

\begin{tabular}{|c|c|}
\hline Characteristic & \\
\hline \multicolumn{2}{|l|}{ Age (years), n (\%) } \\
\hline $15-35$ & $246(62.6)$ \\
\hline $36-50$ & $122(31.0)$ \\
\hline$\geq 5$ I & $25(6.4)$ \\
\hline Age (years), median (IQR) & $32(18-67)$ \\
\hline \multicolumn{2}{|l|}{ Geographical region, n (\%) } \\
\hline Pearl River Delta & $334(85.0)$ \\
\hline Eastern & $15(3.8)$ \\
\hline Western & $23(5.9)$ \\
\hline Northern & $21(5.3)$ \\
\hline \multicolumn{2}{|l|}{ Marital status, n (\%) } \\
\hline Unmarried & $270(68.7)$ \\
\hline Married or cohabiting & $97(24.9)$ \\
\hline Divorced or separated & $26(6.6)$ \\
\hline \multicolumn{2}{|l|}{ Baseline CD4+ T cell count (cells/mm3), n (\%) } \\
\hline$\leq 200$ & $243(61.8)$ \\
\hline$>200$ & $150(38.2)$ \\
\hline $\begin{array}{l}\text { Baseline CD4+ T cell count (cells } / \mathrm{mm} 3 \text { ), median } \\
\text { (IQR) }\end{array}$ & $|4|(\mid-966)$ \\
\hline Years from ACT to ART, median (IQR) & $\begin{array}{l}0.10(0.00 \\
8.16)\end{array}$ \\
\hline Years from ART to DRT, median (IQR) & $\begin{array}{l}1.42(0.50- \\
8.17)\end{array}$ \\
\hline \multicolumn{2}{|l|}{ Initial treatment regimen, $\mathrm{n}(\%)$} \\
\hline ZDV/D4T+3TC+NVP/EFV & $74(18.8)$ \\
\hline $\mathrm{TDF}+3 \mathrm{TC}+\mathrm{EFV} / \mathrm{NVP}$ & $285(72.5)$ \\
\hline $\mathrm{ZDV} / \mathrm{TDF}+3 \mathrm{TC}+\mathrm{LPV} / \mathrm{r}$ & $34(8.7)$ \\
\hline DRT viral load (logl0 copies/mL), median (IQR) & $\begin{array}{l}4.37(3.00 \\
6.69)\end{array}$ \\
\hline
\end{tabular}

Abbreviations: ACT, antibody confirmation test; DRT, drug resistance test; ZDV, Zidovudine; D4T, Stavudine; 3TC, Lamivudine; NVP, Nevirapine; EFV, Efavirenz; $T D F$, Tenofovir disoproxil fumarate; LPV/r, Ritonavir-boosted lopinavir. 
and $62.6 \%(246 / 393)$ of them were 15 to 35 years old. Most of them $(334,85.0 \%)$ were distributed in the Pearl River Delta region and unmarried $(270,68.7 \%)$. The median (range) baseline $\mathrm{CD}^{+} \mathrm{T}$ cell count was 141 (1-966) cells/ $\mathrm{mm}^{3}$, and $61.8 \%(243 / 393)$ of the participants had a baseline $\mathrm{CD}^{+} \mathrm{T}$ cell count of $<200$ cells $/ \mathrm{mm}^{3}$. The median (range) HIV-1 viral load $(\log 10$ copies $/ \mathrm{mL})$ in the drug resistance test was 4.37 (3.00-6.69). The dominant initial treatment regimen was Tenofovir (TDF)+ lamivudine (3TC)+ Efavirenz (EFV)/Nevirapine (NVP) (72.5\%, 285/393).

\section{Distribution of Genotypes}

The ML phylogenetic analyses based on the pol gene regions showed that CRF01_AE was the most common strain circulating among MSM with ART failure in Guangdong Province (47.3\%, 186/393), followed by
CRF07_BC (21.4\%, 84/393), CRF55_01B (21.4\%, 84/ 393), subtype B (5.1\%, 20/393), CRF55_01B (2.3\%, 9/ 393) and others (Figure 1, Table 2).

\section{Drug Resistance Mutations}

Of the 393 MSM patients with ART failure, 69.7\% (274/ 393) had at least one DRM. 55.2\% patients (217/393) harboured NRTI resistance mutations, whereas $67.4 \%$ (265/393) carried NNRTI resistance mutations, with only $0.5 \%(2 / 393)$ PI resistance mutations. Dual-class mutations were present in $53.4 \%$ (210/393) of the patients, with 53.2\% (209/393) having NRTI plus NNRTI resistance mutations and $0.3 \%(1 / 393)$ having PI plus NNRTI resistance mutations. No triple-class mutations were found.

One case $(0.3 \%, 1 / 393)$ carried PI-related DRMs, K20T, M46I, and K43T. Among the NRTI DRMs, M184I/V

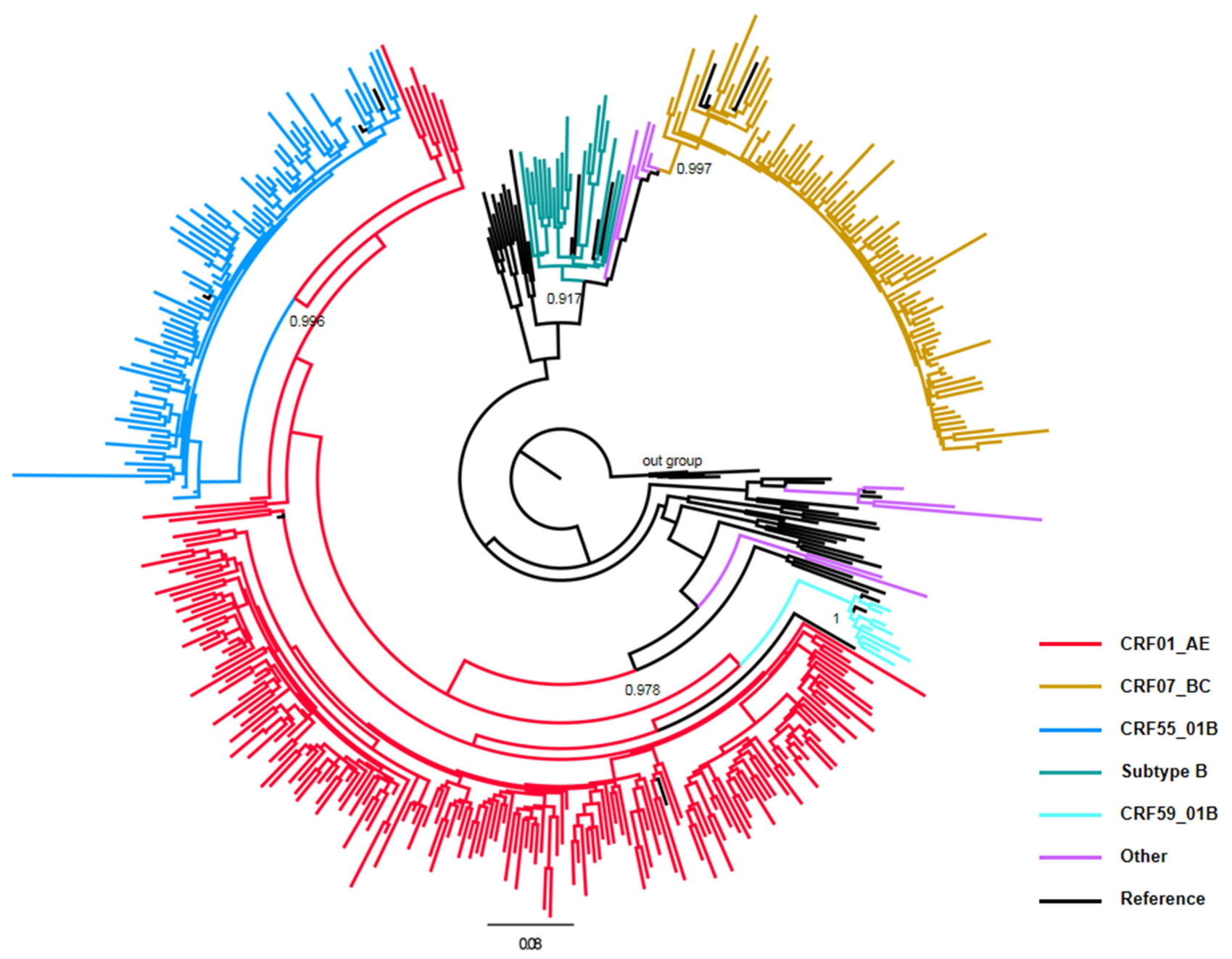

Figure I Distribution characteristics of HIV-I genotypes among MSM with ART failure in Guangdong Province from 2014 to 2019 . The maximum likelihood phylogenetic tree was constructed using PhyML 3.0. The reference sequences, including nine subtypes (A-D, F-H, J and K), CRF0I_AE, CRF07_BC, CRF08_BC, CRF55_0IB, and CRF59_0IB, were downloaded from the Los Alamos HIV Sequence Database (https://www.hiv.lanl.gov/content/index). Different colour lines represent different genotypes. The subtype $\mathrm{H}$ sequences were selected as the outgroup. aLTR values higher than 0.9 were used to identify lineages and are indicated at the corresponding nodes of the tree. 
Table 2 General Information Accompanied with Drug Resistance Among 393 MSM Patients with ART Failure

\begin{tabular}{|c|c|c|c|c|c|}
\hline \multirow[t]{2}{*}{ Characteristics } & \multirow[t]{2}{*}{$\begin{array}{l}\text { HIV Virologic Failure, } \\
\qquad \mathrm{N}=393\end{array}$} & \multirow[t]{2}{*}{$\begin{array}{c}\text { Drug Resistance, } \\
\text { n (\%) }\end{array}$} & \multirow[t]{2}{*}{$\begin{array}{c}P \text { for Fisher Exact } \\
\text { Tests }\end{array}$} & \multicolumn{2}{|c|}{$\begin{array}{c}\text { Multivariate Logistic } \\
\text { Regression }\end{array}$} \\
\hline & & & & $\begin{array}{l}\text { Adjusted OR(95\% } \\
\qquad \mathrm{Cl})\end{array}$ & $P$-value \\
\hline \multicolumn{6}{|l|}{ Age(years) } \\
\hline $15-35$ & 246 & $160(65.0)$ & \multirow[t]{3}{*}{0.656} & & \\
\hline $36-50$ & 122 & $78(63.9)$ & & & \\
\hline$\geq 51$ & 25 & $14(56.0)$ & & & \\
\hline \multicolumn{6}{|l|}{ Occupation } \\
\hline Student & 16 & $8(50.0)$ & \multirow[t]{5}{*}{0.085} & & \\
\hline Farmer & 21 & $8(38.1)$ & & & \\
\hline Employed & 177 & $118(66.7)$ & & & \\
\hline Unemployed & 97 & $63(65.0)$ & & & \\
\hline Unknown & 82 & $55(67.1)$ & & & \\
\hline \multicolumn{6}{|l|}{ Marital status } \\
\hline Unmarried & 270 & $175(64.8)$ & \multirow[t]{3}{*}{0.438} & & \\
\hline Married or cohabiting & 97 & $58(59.8)$ & & & \\
\hline Divorced or separated & 26 & $19(73.1)$ & & & \\
\hline \multicolumn{6}{|l|}{ WHO classification } \\
\hline 1 & 58 & $3 I(53.5)$ & \multirow[t]{4}{*}{0.698} & & \\
\hline II & 56 & $36(64.3)$ & & & \\
\hline III & 244 & $157(64.3)$ & & & \\
\hline IV & 35 & $28(80.0)$ & & & \\
\hline \multicolumn{6}{|l|}{ Geographical region } \\
\hline Pearl River Delta & 334 & $213(63.8)$ & \multirow[t]{4}{*}{0.734} & & \\
\hline Eastern & 15 & II (73.3) & & & \\
\hline Western & 23 & II (47.8) & & & \\
\hline Northern & 21 & $\mid 7(8 \mid .0)$ & & & \\
\hline \multicolumn{6}{|l|}{ Treatment duration(year) } \\
\hline$<1$ & $14 \mid$ & $118(83.7)$ & \multirow[t]{3}{*}{$<0.001$} & 1.000 & \\
\hline $1-3$ & 192 & $113(58.9)$ & & $2.562(1.429-4.590)$ & 0.002 \\
\hline$>3$ & 60 & $21(35.0)$ & & $7.292(3.436-15.476)$ & $<0.001$ \\
\hline \multicolumn{6}{|c|}{ Baseline $C D 4^{+} T$ cell count (cells $/ \mathrm{mm}^{3}$ ) } \\
\hline$\leq 200$ & 243 & $190(78.2)$ & \multirow[t]{2}{*}{$<0.001$} & 1.000 & \\
\hline$>200$ & 150 & $62(4 \mid .3)$ & & $3.896(2.367-6.413)$ & $<0.001$ \\
\hline
\end{tabular}


Table 2 (Continued).

\begin{tabular}{|c|c|c|c|c|c|}
\hline \multirow[t]{2}{*}{ Characteristics } & \multirow[t]{2}{*}{$\begin{array}{l}\text { HIV Virologic Failure, } \\
\qquad \mathrm{N}=393\end{array}$} & \multirow[t]{2}{*}{$\begin{array}{c}\text { Drug Resistance, } \\
\text { n (\%) }\end{array}$} & \multirow[t]{2}{*}{$\begin{array}{c}P \text { for Fisher Exact } \\
\text { Tests }\end{array}$} & \multicolumn{2}{|c|}{$\begin{array}{c}\text { Multivariate Logistic } \\
\text { Regression }\end{array}$} \\
\hline & & & & $\begin{array}{c}\text { Adjusted OR(95\% } \\
\text { Cl) }\end{array}$ & $P$-value \\
\hline \multicolumn{6}{|l|}{ Viral load $(\log 10)$} \\
\hline $3 \sim$ & 134 & $76(56.7)$ & \multirow[t]{3}{*}{0.502} & & \\
\hline $4 \sim$ & 180 & $118(65.6)$ & & & \\
\hline$\geq 5$ & 79 & $58(73.4)$ & & & \\
\hline \multicolumn{6}{|l|}{ Genotypes } \\
\hline Subtype B & 20 & II (55.0) & \multirow[t]{6}{*}{0.040} & 1.000 & \\
\hline CRFOI_AE & 186 & $|3|(70.4)$ & & $0.667(0.235-1.894)$ & 0.447 \\
\hline CRF07_BC & 84 & $30(35.7)$ & & $2.116(0.702-6.376)$ & 0.183 \\
\hline CRF55_OIB & 84 & $68(81.0)$ & & $0.308(0.097-0.980)$ & 0.046 \\
\hline CRF59_0IB & 9 & $4(44.4)$ & & $1.977(0.355-11.026)$ & 0.437 \\
\hline Others & 10 & $8(80.0)$ & & $0.348(0.047-2.584)$ & 0.302 \\
\hline
\end{tabular}

$(42.2 \%, 166 / 393)$ was the most frequent, followed by K65R $(27.2 \%, 107 / 393)$. The most common NRTI mutation pattern was $\mathrm{M} 184 \mathrm{~V}+\mathrm{K} 65 \mathrm{R}$, with a frequency of $15.0 \%$ (59/ 393). The major NNRTI DRMs were V179D/E (37.9\%, 149/393), V106I/M (25.7\%, 101/393), and K103N/Q $(25.2 \%, 99 / 393)$ (Table 3$)$.

\section{Resistance to ART}

252 out of 393 MSM (64.1\%) were resistant to at least one drug (Table 2). Among them, 244 individuals (96.8\%) harboured mutations conveying high-level resistance to NRTIs or NNRTIs. 198 (78.6\%) individuals expressed dual resistance to NRTIs and NNRTIs, whereas 13 $(5.2 \%)$ and $33(13.1 \%)$ ones expressed single resistance to NRTIs and NNRTIs, respectively. Fisher's exact tests showed significant differences in drug resistance rates in terms of different treatment durations, baseline $\mathrm{CD}^{+}$ $\mathrm{T}$ cell counts, and genotypes of HIV-1 $(\mathrm{P}<0.05)$ (Table 2). The patients with a treatment duration of less than one year had the highest drug resistance rate $(83.7 \%$, $118 / 141)$, compared with the other group; the patients with a baseline $\mathrm{CD}^{+} \mathrm{T}$ cell count $\leq 200$ cells $/ \mathrm{mm}^{3}$ had a higher drug resistance rate $(78.2 \%, 190 / 243)$ than the patients with a baseline $\mathrm{CD}^{+} \mathrm{T}$ cell count $>200$ cells $/ \mathrm{mm}^{3}$; and the CRF55_01B patients had the highest drug resistance rate $(81.0 \%, 68 / 84)$ (Table 2$)$.

\section{Genetic Transmission Networks of MSM with ART Failure}

MSM with subtype B, CRF01_AE, CRF07_BC, CRF55_01B, and CRF59_01B pol sequences were identified and used for genetic transmission network analysis. For different genotypes, transmission networks were constructed with different genetic distance thresholds to identify the maximum number of clusters.

Of the 20 subtype B pol sequences, no sequence fell into a cluster at a genetic distance threshold from 0.005 to 0.015. Among 186 CRF01_AE pol sequences, 27 (14.5\%) sequences fell into clusters at a genetic distance threshold of 0.015 , resulting in 8 clusters ranging in size from 2 to 8 (Figure 2A). Of 84 CRF07_BC pol sequences, 33 (39.3\%) sequences fell into clusters at a genetic distance threshold of 0.013 , resulting in 3 clusters ranging in size from 2 to 29 (Figure 2B). Of 84 CRF55_01B pol sequences, 52 $(61.9 \%)$ sequences fell into 3 clusters with sizes of 2 and 48 at a genetic distance of threshold 0.015 (Figure 2C). Of the 9 CRF59_01B pol sequences, 3 (33.3\%) sequences fell into 1 cluster at a genetic distance threshold of 0.015 (Figure 2D)

Among clusters for CRF01_AE pol sequences, 17 MSM (63.0\%) were found to have only one linked individual, and $10(37.0 \%)$ had $\geq 2$ linked individuals. Among 
Table 3 The Number and Prevalence (\%) of Drug Resistance Mutations Among MSM with ART Failure

\begin{tabular}{|c|c|c|c|c|c|c|c|}
\hline \multirow[t]{2}{*}{ DRM } & Total & B & CRFOI_AE & CRF07_BC & CRF55_0IB & CRF59_0IB & Others \\
\hline & $N=393$ & $\mathbf{N}=\mathbf{2 0}$ & $N=186$ & $\mathbf{N}=84$ & $N=84$ & $N=9$ & $N=10$ \\
\hline \multicolumn{8}{|l|}{ PI } \\
\hline M46I & $\mathrm{I}(0.3)$ & - & - & $\mathrm{I}(\mathrm{I} .2)$ & - & - & - \\
\hline $\mathrm{K} 43 \mathrm{~T}$ & $\mathrm{I}(0.3)$ & - & - & $\mathrm{I}(\mathrm{I} .2)$ & - & - & - \\
\hline K20T & $I(0.3)$ & - & - & - & $\mathrm{I}(\mathrm{I} .2)$ & - & - \\
\hline \multicolumn{8}{|l|}{ NRTI } \\
\hline MI84I/V & $166(42.2)$ & $8(40.0)$ & $85(45.7)$ & $20(23.8)$ & $45(53.6)$ & $4(44.4)$ & $4(40.0)$ \\
\hline K65R & $107(27.2)$ & $4(20.0)$ & $57(30.7)$ & II(I3.I) & $30(35.7)$ & $I(I I . I)$ & $4(40.0)$ \\
\hline $\mathrm{K} 70 \mathrm{E} / \mathrm{Q} / \mathrm{R} / \mathrm{T}$ & $53(13.5)$ & $4(20.0)$ & $25(13.4)$ & $6(7.1)$ & $15(17.9)$ & $I(I I . I)$ & $2(20.0)$ \\
\hline YII5F & $5 I(13.0)$ & $2(10.0)$ & $33(17.7)$ & $3(3.6)$ & $10(11.9)$ & - & $3(30.0)$ \\
\hline D67G/N & $4 I(10.4)$ & $4(20.0)$ & $25(13.4)$ & $7(8.3)$ & $4(4.8)$ & $I(I I . I)$ & - \\
\hline $\mathrm{A} 62 \mathrm{~V}$ & $22(5.6)$ & $2(10.0)$ & $12(6.5)$ & - & $6(7.1)$ & $I(I I . I)$ & $I(I 0.0)$ \\
\hline L74I/V & $19(4.8)$ & - & $12(6.5)$ & $3(3.6)$ & $4(4.8)$ & - & - \\
\hline K219E/Q & $16(4.1)$ & $I(5.0)$ & $7(3.8)$ & $\mathrm{I}(\mathrm{I} .2)$ & $7(8.3)$ & - & - \\
\hline $\mathrm{T} 2 \mathrm{I} 5 \mathrm{~F} / \mathrm{I} / \mathrm{Y}$ & $14(3.6)$ & $I(5.0)$ & $6(3.2)$ & $\mathrm{I}(\mathrm{I} .2)$ & $6(7.1)$ & - & - \\
\hline M4IL & $12(3.1)$ & - & $6(3.2)$ & $2(2.4)$ & $4(4.8)$ & - & - \\
\hline V75I/M & $12(3.1)$ & - & $9(4.8)$ & - & $3(3.6)$ & - & - \\
\hline L2IOW & $2(0.5)$ & - & - & $\mathrm{I}(\mathrm{I} .2)$ & $\mathrm{I}(\mathrm{I} .2)$ & - & - \\
\hline F77L & $\mathrm{I}(0.3)$ & - & $\mathrm{I}(0.5)$ & - & - & - & - \\
\hline T69N & $\mathrm{I}(0.3)$ & - & $\mathrm{I}(0.5)$ & - & - & - & - \\
\hline \multicolumn{8}{|l|}{ NNRTI } \\
\hline VI79D/E & $149(37.9)$ & $5(25.0)$ & $45(24.2)$ & $9(10.7)$ & $84(100.0)$ & $I(I I . I)$ & $5(50.0)$ \\
\hline VI06I/M & $101(25.7)$ & $3(15.0)$ & $6 I(32.8)$ & $12(14.3)$ & $20(23.8)$ & $2(22.2)$ & $3(30.0)$ \\
\hline $\mathrm{K} / 03 \mathrm{~N} / \mathrm{Q}$ & $99(25.2)$ & $6(30.0)$ & $40(2 \mid .5)$ & $9(10.7)$ & $39(46.4)$ & $3(33.3)$ & $2(20.0)$ \\
\hline GI90A/S & $64(16.3)$ & $4(20.0)$ & $5 I(27.4)$ & $4(4.8)$ & $4(4.8)$ & - & $\mathrm{I}(\mathrm{I} 0.0)$ \\
\hline YI8IC & $64(16.3)$ & $2(10.0)$ & $34(18.3)$ & $5(6.0)$ & $19(22.6)$ & $I(I I . I)$ & $3(30.0)$ \\
\hline YI88C/H/L & $28(7.1)$ & $2(10.0)$ & II(5.9) & $2(2.4)$ & $12(14.3)$ & - & $I(10.0)$ \\
\hline $\mathrm{KIOIE} / \mathrm{H} / \mathrm{P}$ & $26(6.6)$ & - & $20(10.8)$ & $5(6.0)$ & $\mathrm{I}(\mathrm{I} .2)$ & - & - \\
\hline $\mathrm{F} 227 \mathrm{~L}$ & $26(6.6)$ & - & $14(7.5)$ & $9(10.7)$ & $3(3.6)$ & - & - \\
\hline $\mathrm{H} 22 \mathrm{IY}$ & $19(4.8)$ & $I(5.0)$ & $12(6.5)$ & - & $5(6.0)$ & - & $I(I 0.0)$ \\
\hline $\mathrm{EI} 38 \mathrm{~A} / \mathrm{G}$ & $13(3)$. & $I(5.0)$ & $2(I . I)$ & $\mathrm{I}(1.2)$ & $9(10.7)$ & - & - \\
\hline $\mathrm{M} 230 \mathrm{~L}$ & $\mathrm{II}(2.8)$ & - & $7(3.8)$ & - & $4(4.8)$ & - & - \\
\hline V108I & $10(2.5)$ & - & $5(2.7)$ & - & $4(4.8)$ & - & $I(10.0)$ \\
\hline
\end{tabular}


Table 3 (Continued).

\begin{tabular}{|c|c|c|c|c|c|c|c|}
\hline \multirow[t]{2}{*}{ DRM } & Total & B & CRFOI_AE & CRF07_BC & CRF55_0IB & CRF59_OIB & Others \\
\hline & $N=393$ & $\mathbf{N}=\mathbf{2 0}$ & $N=186$ & $N=84$ & $\mathbf{N}=84$ & $N=9$ & $N=10$ \\
\hline $\mathrm{P} 225 \mathrm{H}$ & $8(2.0)$ & $2(10.0)$ & $4(2.2)$ & - & $2(2.4)$ & - & - \\
\hline LIOOI & $7(1.8)$ & $2(10.0)$ & $\mathrm{I}(0.5)$ & - & $3(3.6)$ & - & $I(I 0.0)$ \\
\hline A98G & $5(1.3)$ & - & $3(1.6)$ & - & $2(2.4)$ & - & - \\
\hline $\mathrm{K} 238 \mathrm{~T}$ & $3(0.8)$ & - & $\mathrm{I}(0.5)$ & - & $2(2.4)$ & - & - \\
\hline L234I & $2(0.5)$ & - & $2(I .1)$ & - & - & - & - \\
\hline
\end{tabular}

Note: The major mutations were denoted by bolding.

clusters for CRF07_BC pol sequences, 16 MSM (48.5\%) were found to have only one linked individual, and 17 (51.5\%) had $\geq 2$ linked individuals. Among the clusters for CRF55_01B pol sequences, 17 MSM (32.7\%) were found to have only one linked individual, and 35 (67.3\%) had $\geq 2$ linked individuals. Of all 15 transmission networks, 69 $(60.0 \%, 69 / 115)$ sequences from patients aged 15 to 35 years old were dispersed among $73.3 \%$ (11/15) of transmission networks, and $59(51.3 \%, 59 / 115)$ sequences from patients with drug resistance were dispersed among $86.7 \%$ (13/15) of transmission networks (Figure 2).

There were no significant differences between genetic linkages in terms of age, occupation, marital status, WHO classification, geographical region, treatment duration, baseline $\mathrm{CD}^{+} \mathrm{T}$ cell count, viral $\operatorname{load}(\log 10)$ in ART failure, or individuals who showed or did not show drug resistance after ART failure (Table 4).

\section{Discussion}

HIV epidemics in Guangdong have expanded beyond injection drug users and grown into sexual populations, including MSM and heterosexual contact, since 2013. ${ }^{15}$ The occurrence of HIV-1 acquired drug resistance becomes a critical clinical and public health issue and will lead to ART failure. This study analysed the prevalence of resistant variants and linking-associated factors for genetic transmission networks of MSM with ART failure in Guangdong, providing implications for the management of ART.

The results show that CRF01_AE (47.3\%) and CRF07_BC (21.4\%) were the dominant circulating HIV1 strains in MSM with ART failure in Guangdong. A recent nationwide survey in China revealed that CRF01_AE and CRF07_BC accounted for more than $80 \%$ of HIV-1 infections among MSM in nine major
Chinese cities, ${ }^{16}$ which was consistent with our results. CRF55_01B is the CRF01_AE/B recombinant strain originating from MSM in Shenzhen, Guangdong, and was first reported in 2013. ${ }^{17,18}$ In the present study, CRF55_01B was the third most dominant circulating genotype, accounting for $21.4 \%$, higher than the $8.5 \%$ in Guangdong in $2013,{ }^{19} 9.2 \%$ in Shenzhen in 2006$2013,{ }^{18}$ and $10.7 \%$ in Guangzhou in $2018 .^{20}$

The overall prevalence of drug resistance was $66.7 \%$ in MSM with ART failure in Guangdong, which was similar to that in Yunnan $(66.27 \%$ in MSM with ART failure from 2014 to 2019$)^{21}$ and relatively higher than those in other areas of China, such as Sichuan $(50.7 \%$ in MSM with ART failure from 2011 to 2017) $)^{22}$ and Hunan (41.3\% in MSM with ART failure from 2012 to 2017). ${ }^{23}$

M184I/V (42.2\%) was the most prevalent NRTIassociated mutation, which might be caused by the frequent use of 3TC in ART-experienced patients. ${ }^{24}$ This caused high-level resistance to $3 \mathrm{TC}$ and Emtricitabine (FTC) and low-level resistance to Abacavir (ABC) and Didanosine (DDI). In contrast, M184I/V increased susceptibility to Zidovudine (AZT), Stavudine (D4T) and TDF and slowed the emergence of AZT, D4T, and TDF resistance. K65R (27.2\%) is the second most common NRTI-associated resistance mutation and has only a relatively low level of reduced drug susceptibility. A high frequency of K65R plus M184V/I (15.0\%) was observed in this study, and K65R plus M184 V/I appeared sufficient to abrogate the NRTI activity of a regimen comprising $\mathrm{ABC}$, TDF or D4T. ${ }^{25}$ As a consequence, it should be notified that more attention should be reinforced to the possibility of transmitted drug resistance, when preexposure prophylaxis (PrEP) was administered in this population. In our study, V179E/D was the most common NNRTI-associated mutation, accounting for $37.9 \%$. 
A

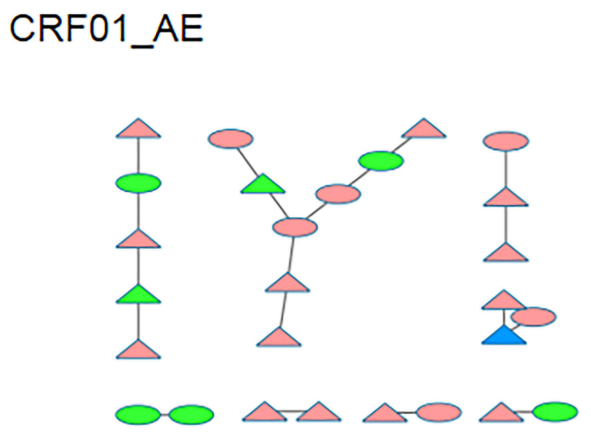

B

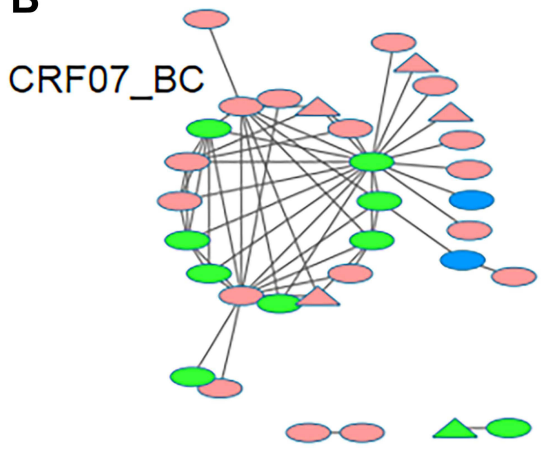

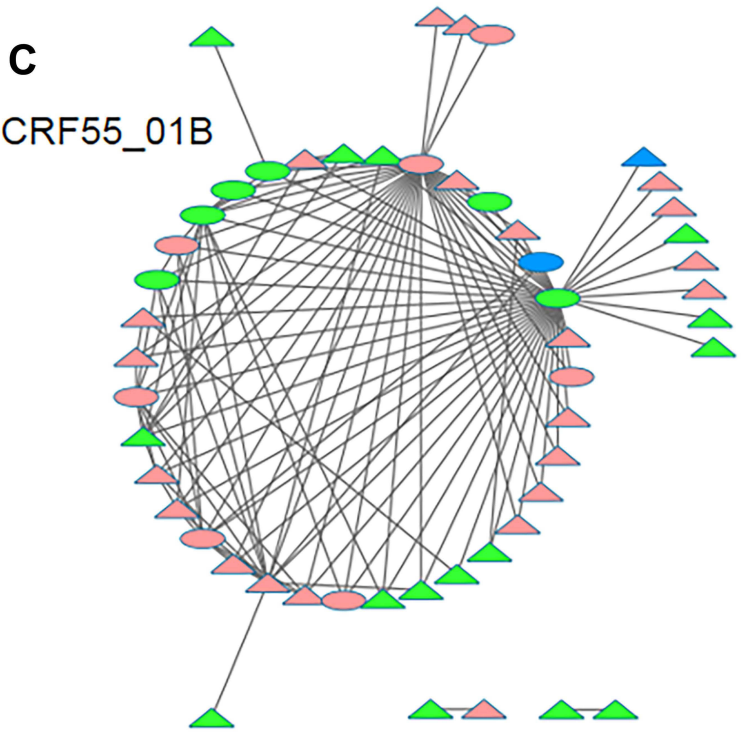

D

CRF59_01B

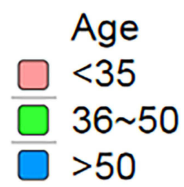

\section{$\triangle$ Drug resistance \\ Non-drug resistance}

Figure 2 Age- and drug-resistance-associated genetic transmission networks of different genotype sequences. The networks were constructed using Cytoscape with a pairwise genetic distance analysed by using HyPhy software. A total of 27 CRF0I AE sequences (A), 33 CRF07_BC sequences (B), 52 CRF55 0 IB sequences (C), and 3 CRF59_0IB sequences (D) were used for genetic transmission network analysis, and the largest cluster had 48 sequences. Different colours and shapes represent sequences from different age groups or those showing drug resistance or not, respectively.

V179E is a nonpolymorphic mutation weakly selected by NVP and EFV. ${ }^{26}$ V179D reduces NVP and EFV susceptibility by 2- to 5-fold and reduces ETR and RPV susceptibility by 2- to 3-fold, respectively. ${ }^{27}$ V179E/D does not appear to reduce the virological response to a first-line EFV-containing regimen. ${ }^{15}$ V106I/M, K103N/Q, G190A/ $\mathrm{S}$, and Y181C were the major NNRTI-associated mutations, similar to those in other cities in China and other countries, ${ }^{23,28-31}$ and showed broad-spectrum resistance possibly caused by the wide use of NNRTIs.

The parameters examined in this study had no significant effect on drug resistance, leading to treatment failure except treatment duration, baseline $\mathrm{CD} 4^{+}$cell count, and genotype
$(\mathrm{P}<0.001,<0.001$, and 0.040 , respectively). Virologic failure without resistance is most often the result of inadequate drug concentrations caused by poor adherence or drug-drug interactions. ${ }^{32}$ In the present study, patients with a treatment duration below one year $(83.7 \%)$ or $\mathrm{CD}^{+}$cell count below 200 cells $/ \mathrm{mm}^{3}(78.2 \%)$ had a higher drug resistance rate than other groups in terms of the corresponding factors. More attention should be paid to differentiate between adherence- and resistance-driven failures, which are not mutually exclusive. In line with the evidence that different frequencies of DRMs among HIV-1 subtypes circulate among drug-naive and treated individuals in China, ${ }^{33}$ there were different drug resistance rates among different genotypes in this study (Table 2). 


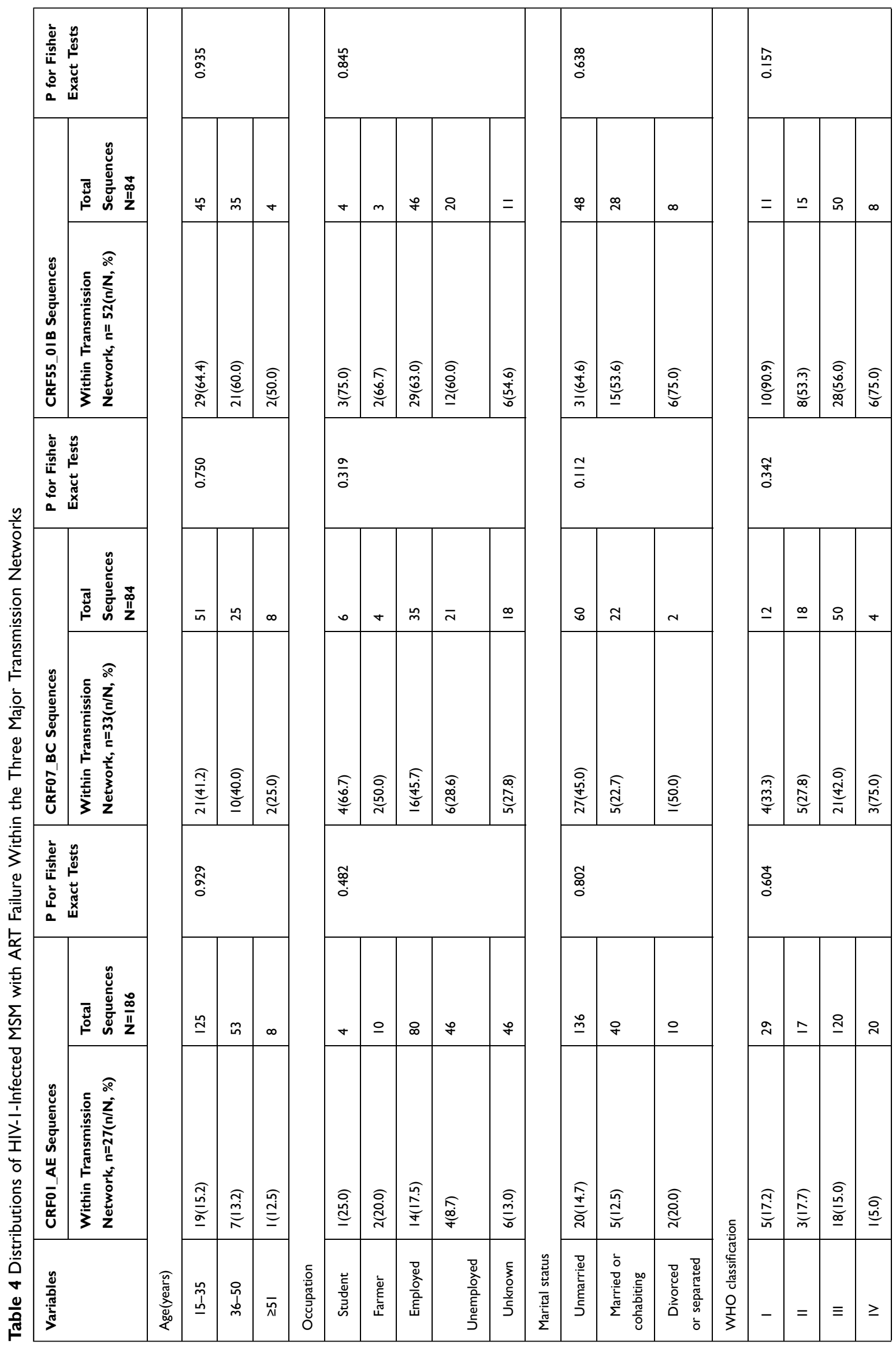




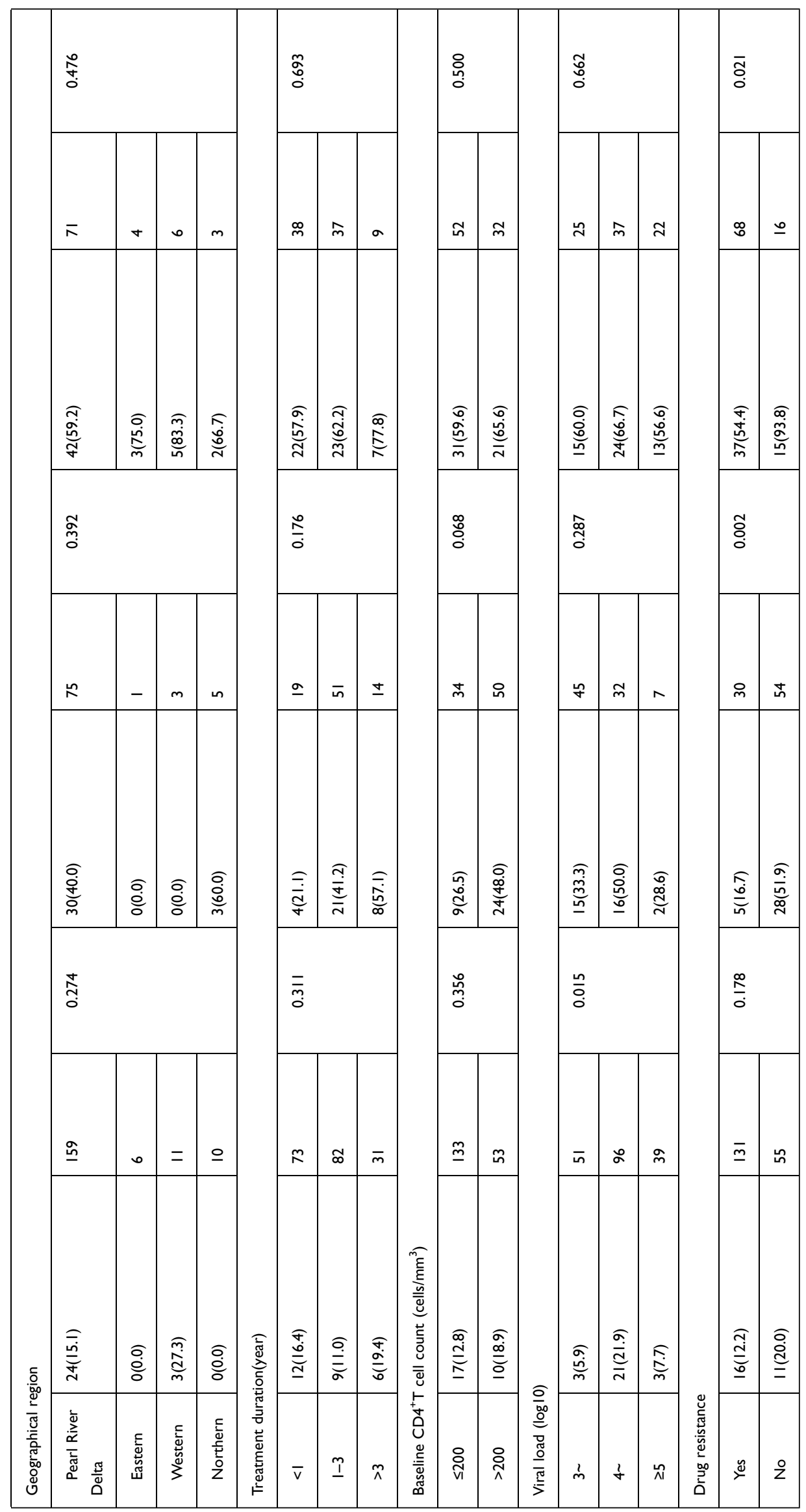


The genetic distance cut-off value for constructing the transmission network differs among sequences belonging to different subtypes. ${ }^{34}$ In the present study, genetic distances $\leq 0.013$ and $\leq 0.015$ were set as cut-off values for CRF07_BC and CRF01_AE, CRF55_01B, or CRF59_01B, respectively. The results showed that most (70.4\% for CRF01_AE, 63.6\% for CRF07_BC, and 55.8\% for CRF55_01B) of the MSM in transmission networks were concentrated at ages below 35 years old. Moreover, approximately $54.8 \%(63 / 115)$ of MSM had more than 2 potential transmission partners. Individuals with multiple links could have potentially higher transmission risk. ${ }^{22}$ Therefore, more attention should be given to younger HIV-1-infected MSM with multiple links.

\section{Conclusions}

Our study focused on the genotype diversity, drug resistance and transmission networks among MSM with ART failure in Guangdong Province from 2014 to 2019 and identified CRF01_AE, CRF07_BC and CRF55_01B as the main strains circulating among MSM with ART failure. Drug resistance mutations more frequently occurred in NNRTIs. Transmission network analysis revealed a complex transmission pattern, and more attention should be paid to younger HIV-1-infected MSM with multiple links.

\section{Ethics Approval}

This study was approved by the institutional review board of the Guangzhou Eighth People's Hospital. All participants in this study provided written informed consent.

\section{Acknowledgments}

The authors thank Prof. Xiang He from Guangdong Provincial Institute of Public Health, and Prof. Ruolei Xin from Beijing Center for Disease Prevention and Control, for their comments on this paper.

\section{Funding}

This study was supported by grants from the National Major Scientific and Technological Special Project during the 13th Five-Year Plan Period (2017ZX10202101-003), the Joint-Innovative Program in Healthcare for Special Scientific Research Projects of Guangzhou (201803040002), and Guangzhou Science and Technology Plan Project (202002030028).

\section{Disclosure}

The authors declare no conflicts of interest for this work.

\section{References}

1. NCAIDS N, China CDC. Update on the AIDS/STD epidemic in China in August, 2018. Chin J AIDS STD. 2018;24:965. doi:10.13419/j.cnki.aids.2018.10.01

2. Boyd AT, Oboho I, Paulin H, et al. Addressing advanced HIV disease and mortality in global HIV programming. AIDS Res Ther. 1999;17 (1):40. doi:10.1186/s12981-020-00296-x

3. Hingankar NK, Thorat SR, Deshpande A, et al. Initial virologic response and HIV drug resistance among HIV-infected individuals initiating firstline antiretroviral therapy at 2 clinics in Chennai and Mumbai, India. Clin Infect Dis. 2012;54(supp1_4):S348-54. doi: $10.1093 / \mathrm{cid} / \mathrm{cis} 005$

4. Beyrer C, Abdool Karim Q. The changing epidemiology of HIV in 2013. Curr Opin HIV AIDS. 2013;8:306-310. doi:10.1097/ $\mathrm{COH} .0 \mathrm{~b} 013 \mathrm{e} 328361 \mathrm{f} 53 \mathrm{a}$

5. Li Z, Liao L, Feng Y, et al. Trends of HIV subtypes and phylogenetic dynamics among young men who have sex with men in China, 20092014. Sci Rep. 2015;5(1):16708. doi:10.1038/srep16708

6. Li Y, Lin P, Wang Y, et al. HIV/AIDS surveillance in Guangdong Province, 2000-2013. South Chin J Prev Med. 2015;41:101-106. doi:10.13217/j.scjpm.2015.0101

7. Zhang J, Guo Z, Pan X, et al. Highlighting the crucial role of Hangzhou in HIV-1 transmission among men who have sex with men in Zhejiang, China. Sci Rep. 2017;7(1):13892. doi:10.1038/ s41598-017-14108-2

8. Struck D, Lawyer G, Ternes AM, et al. COMET: adaptive context-based modeling for ultrafast HIV-1 subtype identification. Nucleic Acids Res. 2014;42(18):e144. doi:10.1093/nar/gku739

9. Guindon S, Dufayard JF, Lefort V, et al. New algorithms and methods to estimate maximum-likelihood phylogenies: assessing the performance of PhyML 3.0. Syst Biol. 2010;59(3):307-321. doi:10.1093/ sysbio/syq010

10. Anisimova M, Gascuel O, Sullivan J. Approximate likelihood-ratio test for branches: a fast, accurate, and powerful alternative. Syst Biol. 2006;55(4):539-552. doi:10.1080/10635150600755453

11. Tang MW, Liu TF, Shafer RW. The HIVdb system for HIV-1 genotypic resistance interpretation. Intervirology. 2012;55(2):98-101. doi: $10.1159 / 000331998$

12. Stanford University HIV drug resistance database. Major HIV-1 Drug Resistance Mutations; 2020. Available from: https://cms.hivdb.org/ prod/downloads/resistance-mutation-handout/resistance-mutationhandout.pdf. Accessed July 22, 2021.

13. Wertheim JO, Kosakovsky Pond SL, Forgione LA, et al. Social and genetic networks of HIV-1 transmission in New York City. PLoS Pathog. 2017;13(1):e1006000. doi:10.1371/journal.ppat.1006000

14. Hassan AS, Pybus OG, Sanders EJ, et al. Defining HIV-1 transmission clusters based on sequence data. AIDS. 2017;31(9):1211-1222. doi:10.1097/QAD.0000000000001470

15. Yu G, Li Y, Huang X, et al. Genetic diversity and drug resistance of HIV-1 CRF55_01B in Guangdong, China. Curr HIV Res. 2020;18 (3):210-218. doi:10.2174/1570162X18666200415140652

16. Han X, An M, Zhang M, et al. Identification of 3 distinct HIV-1 founding strains responsible for expanding epidemic among men who have sex with men in 9 Chinese cities. J Acquir Immune Defic Syndr. 2013;64(1):16-24. doi:10.1097/QAI.0b013e3182932210

17. Han X, An M, Zhang W, et al. Genome sequences of a novel HIV-1 circulating recombinant form, CRF55_01B, identified in China. Genome Announc. 2013;1:e0050-12. doi:10.1128/genomeA.00050-12

18. Zhao J, Cai W, Zheng C, et al. Origin and outbreak of HIV-1 CRF55_01B among MSM in Shenzhen, China. J Acquir Immune Defic Syndr. 2014;66(3):e65-7. doi:10.1097/QAI.0000000000000144

19. Zhou PP, Yu G, Kuang YQ, et al. Rapid and complicated HIV genotype expansion among high-risk groups in Guangdong Province, China. BMC Infect Dis. 2019;19(1):185. doi:10.1186/ s12879-019-3788-7 
20. Lan Y, He X, Li L, et al. Complicated genotypes circulating among treatment naïve HIV-1 patients in Guangzhou, China. Infect Genet Evol. 2021;87:104673. doi:10.1016/j.meegid.20 20.104673

21. Ruan WQ, Liu JF, Zhang M, et al. Genotypic drug resistance of HIV-infected MSM who failed in antiviral therapy in Yunnan Province. Prev Med. 2020;3(2):987-991+995. doi:10.19485/j.cnki. issn2096-5087.2020.10.004

22. Yuan D, Du Z, Zhou J, et al. HIV-1 subtype diversity, drug resistance, and genetic transmission networks in men who have sex with men with virologic failure in antiretroviral therapy in Sichuan, China, 2011 to 2017. Medicine (Baltimore). 2019;98(43):e17585. doi:10.1097/MD.0000000000017585

23. Zou X, He J, Zheng J, et al. Prevalence of acquired drug resistance mutations in antiretroviral- experiencing subjects from 2012 to 2017 in Hunan Province of central South China. Virol J. 2020;17(1):38. doi:10.1186/s12985-020-01311-3

24. Vannappagari V, Ragone L, Henegar C, et al. Prevalence of pretreatment and acquired HIV-1 mutations associated with resistance to lamivudine or rilpivirine: a systematic review. Antivir Ther. 2019;24 (6):393-404. doi:10.3851/IMP3331

25. Xu HT, Martinez-Cajas JL, Ntemgwa ML, et al. Effects of the K65R and $\mathrm{K} 65 \mathrm{R} / \mathrm{M} 184 \mathrm{~V}$ reverse transcriptase mutations in subtype C HIV on enzyme function and drug resistance. Retrovirology. 2009;6(1):14. doi:10.1186/1742-4690-6-14

26. Rhee SY, Gonzales MJ, Kantor R, et al. Human immunodeficiency virus reverse transcriptase and protease sequence database. Nucleic Acids Res. 2003;31(1):298-303. doi:10.1093/nar/gkg100

27. Melikian GL, Rhee SY, Varghese V, et al. Non-nucleoside reverse transcriptase inhibitor (NNRTI) cross-resistance: implications for preclinical evaluation of novel NNRTIs and clinical genotypic resistance testing. J Antimicrob Chemother. 2014;69(1):12-20. doi:10. 1093/jac/dkt316
28. Sno R, Labadie-Bracho MY, Grünberg MG, et al. First assessment of acquired HIV-1 drug resistance and mutation patterns in suriname. AIDS Res Hum Retroviruses. 2021;37(7):557-565. doi:10.1089/ AID.2020.0194

29. Omooja J, Nannyonjo M, Sanyu G, et al. Rates of HIV-1 virological suppression and patterns of acquired drug resistance among fisherfolk on first-line antiretroviral therapy in Uganda. $J$ Antimicrob Chemother. 2019;74(10):3021-3029. doi:10.1093/jac/dkz261

30. Li T, Qian F, Yuan T, et al. Drug resistance mutation profiles of the drug-naïve and first-line regimen-treated HIV-1-infected population of Suzhou, China. Virol Sin. 2017;32(4):271-279. doi:10.1007/ s12250-017-4002-y

31. Zuo L, Liu K, Liu H, et al. Trend of HIV-1 drug resistance in China: a systematic review and meta-analysis of data accumulated over 17 years (2001-2017). EClinicalMedicine. 2020;18:100238. doi:10.10 16/j.eclinm.2019.100238

32. McCluskey SM, Siedner MJ, Marconi VC. Management of virologic failure and HIV drug resistance. Infect Dis Clin North Am. 2019;33 (3):707-742. doi:10.1016/j.idc.2019.05.004

33. Sui H, Gui T, Jia L, et al. Different frequencies of drug resistance mutations among HIV-1 subtypes circulating in China: a Comprehensive Study. PLoS One. 2014;9(3):e91803. doi:10.1371/ journal.pone.0091803

34. Zhang D, Wu J, Zhang Y, et al. Genetic characterization of HIV-1 epidemic in Anhui Province, China. Virol J. 2020;17(1):17. doi:10.1186/s12985-020-1281-y
Infection and Drug Resistance

\section{Publish your work in this journal}

Infection and Drug Resistance is an international, peer-reviewed openaccess journal that focuses on the optimal treatment of infection (bacterial, fungal and viral) and the development and institution of preventive strategies to minimize the development and spread of resistance. The journal is specifically concerned with the epidemiology of
Dovepress

antibiotic resistance and the mechanisms of resistance development and diffusion in both hospitals and the community. The manuscript management system is completely online and includes a very quick and fair peerreview system, which is all easy to use. Visit http://www.dovepress.com/ testimonials.php to read real quotes from published authors. 